entirely supports the validity of Darwin's theory, in the locality selected as a test.

Oxford, February 20.

Edward B. Poulton.

\section{The Accuracy of the Principal Triangulation of the United Kingdom.}

THERE was some discussion of this question at the last meeting of the British Association, and an investigation by Capt. H. St. G. L. Winterbotham has been published by the Ordnance Survey (Professional Papers, new series, No. 2). It appears, however, that the measurement of Lossiemouth Base, valuable as it was, did not definitely decide the question, but that a moderate amount of further computation would do so.

(I) It will be generally admitted that statistical evidence as to the precision of any kind of observation is of no great value unless it is based on a large population. For example, if we may assume the principal triangulation to have been all executed by similar observers under similar conditions, and no unjustifiable rejections to have been made, we may accept with great confidence the probable error of $\pm I \cdot 23^{\prime \prime}$ computed from the closure of $55^{2}$ triangles. But nowadays one would not think of estimating the precision of a baseline by the discrepancy between two measurements of it, though one might reject the measurements if they did not agree as well as was to be expected from the known usual probable error of a measurement. Thus discussion of a large population of discrepancies gives a good estimate of the probable accidental error of a measurement, but a small population only fixes a lower limit to that probable error. Now we have only a population of three independent discrepancies between the four bases of Lough Foyle, Salisbury Plain, Lossiemouth, and Paris, and even if it is brought up to six by the inclusion of the three bases measured with steel chains, yet it must be considered a very small population upon which to base any estimate of precision.

(2) But the original question, to what extent the strength of the figure compensates for the large probable error of an angle, is not necessarily a question for experiment, but it is essentially a question for computation. Given the probable error of an angle in a block of triangulation adjusted rigorously by least squares, if it is required to find the probable error of the distance from any point of it to any other point, the first step is to express the unknown error in that length as a linear function of the unknown errors in a number of independent angles (i.e. two angles of each triangle in a chain of independent triangles stretching from one point to the other, and in another chain stretching from one of the points to the nearest base). It then remains only to add another column to the least square computation by the method described in Wright and Hayford's "Adjustment of Observations," paragraph I23. May I venture to suggest that if two or three such cases could be worked out for strong figures in the United Kingdom, and for comparison two or three cases for weaker figures, either there or elsewhere, it would not only set at rest the immediate question, but would also establish results of great importance for surveyors in general.

T. L. BENNETT.

Computation Office, Egyptian Survey Department.

ONE can but agree with Mr. Bennett in insisting on a large "population" of discrepancies upon which to found a calculation of a probable error, whether it be of the measurement of a base-line or of an angle.

His example of a base-line measurement is not, howNO. 23 I3, VOL. 92$]$ ever, strictly comparable with the investigation in question. In the former a large number of independent measurements must be made from which to deduce the most probable length and the individual discrepancies from this length. In the latter the measured bases may be regarded as errorless compared with the triangulation, and the actual errors can therefore be deduced with surety.

Neglecting the steel chain bases, we have four independent measures upon any one of which the triangulation can be made to depend. These four are widely distributed.

The longest line from base to base is that from the new base at Lossiemouth to the Paris base, and along this line the old steel chain bases add additional proof that no serious errors are inherent in the triangulation.

I agree that, "if we may assume the principal triangulation to have been all executed by similar observers under similar conditions and no unjustifiable rejections to have been made, we may accept with confidence the probable error of $1 \cdot 23^{\prime \prime}$ computed from the closure of 552 triangles," but it must be remembered that this is the probable error of an observed angle. The date of the work makes this a matter of no surprise. The point at issue, however, is not the probable error of an observed angle, but the probable error of an adjusted angle, or, in other words, to find out how far the intricacy of the figure has compensated for the lack of precision of angular measurement.

To say that this question is essentially one for computation is not, to my mind, correct.

The probable error of the ratio of any two sides as derived from the triangulation depends upon the probable error of an adjusted angle. This calculation is possible, but from the complexity of the figure and the intricate system of weighting the angles, impracticable. Moreover, the answer to it would still be the probable, and not the actual, error.

It is, however, possible to pick out of the general figure chains of simple triangles connecting the bases. Supposing that these chains had been the only paths of calculation, and that they had shown the same errors of ratio between the bases as are actually found, we can deduce what the probable error of an observed angle would have been to have effected this result. Such an investigation has been made, and the probable error of an observed angle in these "equivalent" simple chains is $0.85^{\prime \prime}$, or approximately the same as that given by General Ferrero, in his 1892 report, as the mean figure for the probable error of an observed angle in the triangulations of those twenty countries represented on the International Geodetic Association.

Although, therefore, further investigation on the lines advocated by $\mathrm{Mr}$. Bennett would be one of the greatest interest, I do not think that it promises a result commensurate with the time and expense it would entail.

H. S. L. Winterbotham.

\section{Atomic Models}

I AM indebted to $\mathrm{Mr}$. Chalmers for pointing out (NATURE, February I9, p. 687), what I had indeed suspected, that the magnetic moment due to an electron moving in a circular orbit, assuming the angular momentum to be $h / 2 \pi$, is exactly five times the magnetic moment of the magneton. The original value $\left(15.94 \times 10^{-22}\right)$ of the latter quantity, given by Weiss, and quoted in my former letter, was based on the value of Avogadro's constant found by Perrin. If we divide the magnetic moment of the atom gram, I123.5, by the more recent value for Avogadro's constant given by Millikan $\left(60.62 \times \mathrm{IO}^{22}\right)$ we obtain as the magnetic moment of the magneton $18.54 \times \mathrm{ro}^{-22}$, which is exactly 